\title{
Correlation between Serum Lipid Parameters and Interleukin-10 Concentration in Obese Individuals
}

\author{
Soo Jin Kim', Jisun Lim', Ga Eun Nam ${ }^{2, *}$, Hye Soon Park ${ }^{1}$ \\ ${ }^{1}$ Department of Family Medicine, University of Ulsan College of Medicine, Seoul; ${ }^{2}$ Department of Family Medicine, Korea University College of Medicine, Seoul, Korea
}

Background: The role of interleukin-10 (IL-10) in humans is controversial because IL-10 has been proposed to exhibit both pro- and anti-inflammatory effects. We aimed to determine the relationships between the changes in these parameters in obese individuals participating in a weight-reduction program.

Methods: We measured cardiometabolic parameters including lipid profile and serum IL-10 concentration before and after completion of a 12-week weight-reduction program in 63 non-diabetic obese subjects with a body mass index $\geq 27 \mathrm{~kg} / \mathrm{m}^{2}$ who had comorbid hypertension or dyslipidemia. All the participants were provided with individual intervention sessions designed to implement lifestyle modifications and administered 120 mg orlistat three times daily for 12 weeks. The relationships between changes in serum IL-10 concentration and changes in cardiometabolic risk factors were analyzed.

Results: Changes in serum IL-10 concentration were significantly negatively correlated with changes in total cholesterol ( $r=-0.377)$, high-density lipoprotein cholesterol (HDL-C; $r=-0.377)$, and low-density lipoprotein cholesterol ( $L D L-C ; r=-0.278$ ) concentrations. However, there were no correlations between changes in serum IL-10 concentration and changes in other cardiometabolic parameters.

Conclusion: Serum IL-10 concentration can increase as serum total cholesterol decreases. Additional studies are needed to explore the mechanisms linking changes in serum IL-10 with serum LDL-C and HDL-C concentrations.

Key words: Interleukin-10, LDL cholesterol, HDL cholesterol, Weight reduction, Obesity

\author{
Received November 20, 2020 \\ Reviewed March 12, 2021 \\ Accepted March 26, 2021 \\ *Corresponding author \\ Ga Eun Nam \\ (iD) \\ https://orcid.org/0000-0002-6739-9904 \\ Department of Family Medicine, Korea \\ University Anam Hospital, Korea \\ University College of Medicine, \\ 73 Goryeodae-ro, Seongbuk-gu, \\ Seoul 02841, Korea \\ Tel: +82-2-920-5104 \\ Fax: +82-2-928-8083 \\ E-mail:namgaaa@daum.net
}

The first two authors contributed equally to this study.

\section{INTRODUCTION}

Interleukin-10 (IL-10) is a potent anti-inflammatory cytokine secreted by activated monocytes/macrophages and lymphocytes and inhibits the expression of proinflammatory cytokines. ${ }^{1}$ The expression of IL-10 in atherosclerotic plaques is associated with reduction in signs of inflammation. ${ }^{2}$ In addition, low serum IL-10 is associated with metabolic syndrome in obese women, ${ }^{3}$ and IL-10 concentration is positively associated with insulin sensitivity in young healthy adults. ${ }^{4}$ Circulating IL-10 concentration increases after weight reduction in obese humans. ${ }^{5}$ Furthermore, previous studies have demonstrated anti-inflammatory and protective effects of IL-10 against atherosclerosis in acute coronary syndrome ${ }^{6}$ and cerebral infarction. ${ }^{7}$

However, the results of recent studies have complicated the interpretation of the role of IL-10 in humans. Indeed, IL-10 has been proposed to have both inflammatory and anti-inflammatory activities. ${ }^{8}$ High IL-10 concentration has been shown to be a biomarker of poor clinical outcome, reflecting systemic inflammation, in patients with acute coronary syndrome. ${ }^{9}$ In addition, the outcomes of clinical trials of the therapeutic potential of IL-10 for inflammatory diseases have been disappointing. ${ }^{10}$ Finally, serum IL-10 concentration has been shown to decrease after weight loss in obese women. ${ }^{3}$ In addition to the conflicting results of many studies of IL-10 in

Copyright (C) 2021 Korean Society for the Study of Obesity

(a) This is an Open Access article distributed under the terms of the Creative Commons Attribution Non-Commercial License (https://creativecommons.org/licenses/by-nc/4.o/) which permits unrestricted non-commercial use, distribution, and reproduction in any medium, provided the original work is properly cited 
humans, little data have been recorded regarding the dynamic relationships between cardiometabolic risk factors and IL-10 concentration. Therefore, we investigated the relationships between changes in serum IL-10 concentration and changes in cardiometabolic risk factors in non-diabetic obese subjects participating in a 12-week weight-reduction program.

\section{METHODS}

We recruited non-diabetic obese individuals with a body mass index $(\mathrm{BMI}) \geq 27 \mathrm{~kg} / \mathrm{m}^{2}$ who had comorbid hypertension or dyslipidemia and were 20-65 years old. We excluded individuals who had secondary obesity, endocrine disease, malignancy, cardiovascular disease, myocardial infarction, end-stage renal and/or hepatic disease, uncontrolled hypertension, or a history of medication affecting body weight, including diuretics or $\beta$-blockers, within the preceding 3 months. We provided a 12 -week weight-reduction program consisting of individual intervention sessions designed to implement behavioral strategies related to dietary habits and physical activity. The participants were educated to reduce overeating, binge eating, night eating, and alcohol drinking. They were advised to restrict their caloric intake to $\sim 500 \mathrm{kcal}$ less than their usual intake and to consume a diet comprising 60\%-65\% carbohydrates, $20 \%-25 \%$ fats, and $15 \%$ proteins. We advised the participants to engage in regular moderate-intensity low-impact aerobic exercise such as brisk walking, cycling, or swimming. Their diet and physical activity were monitored by clinical dieticians once a month using a diary. All the participants were administered $120 \mathrm{mg}$ orlistat three times daily for 12 weeks. This study was approved by the Institutional Review Board of the Asan Medical Center, Seoul, Korea (IRB No. 2008-0562), and written informed consent was obtained from all the participants.

Blood biochemical parameters were measured using an autoanalyzer (Hitachi-747; Tokyo, Japan). Glycosylated hemoglobin was measured by immunoturbidimetry (Roche, Basel, Switzerland). Fasting insulin (Dianabott, Tokyo, Japan) and adiponectin (Millipore, St. Charles, MO, USA) were measured using radioimmunoassay kits. Homeostasis model assessment-insulin resistance was calculated as fasting insulin $(\mu \mathrm{IU} / \mathrm{mL}) \times$ fasting glucose $(\mathrm{mg} / \mathrm{dL}) /$ 405. ${ }^{11}$ Serum IL-10 was measured by enzyme-linked immunosor- bent assay (Invitrogen, CA, USA) with an intra-assay coefficient of variation of $2.9 \%-4.8 \%$. Comparisons of values before and after weight loss were performed using Wilcoxon signed-rank tests. To evaluate the correlations between changes in serum IL-10 concentration and changes in cardiometabolic risk factors, we calculated correlation coefficients by partial correlation analysis after adjustment for age using IBM SPSS version 21.0 (IBM Corp., Armonk, NY, USA).

\section{RESULTS}

Seventy-six participants were initially enrolled in the study program, and a final total of 63 individuals completed it (dropout rate, $17.1 \%)$. The mean age was 38.2 years, and the proportion of females was $60.3 \%$. Table 1 shows the changes in anthropometric measurements, cardiometabolic variables, and serum IL-10 concentration after weight reduction. The means of BMI before and after intervention were $31.91 \pm 0.34 \mathrm{~kg} / \mathrm{m}^{2}$ and $30.99 \pm 0.34 \mathrm{~kg} / \mathrm{m}^{2}$, respectively $(P<0.001)$. Waist circumference, waist-to-hip ratio, total cholesterol, and high-density lipoprotein cholesterol (HDL-C)

Table 1. Changes in cardiometabolic risk factors and serum IL-10 concentration during weight reduction

\begin{tabular}{|c|c|c|c|c|}
\hline Parameter & Before & After & Difference & $P$ \\
\hline Body weight (kg) & $86.84 \pm 1.51$ & $84.31 \pm 1.45$ & $-2.53 \pm 0.32$ & $<0.001$ \\
\hline $\mathrm{BMI}\left(\mathrm{kg} / \mathrm{m}^{2}\right)$ & $31.91 \pm 0.34$ & $30.99 \pm 0.34$ & $-0.92 \pm 0.12$ & $<0.001$ \\
\hline WC (cm) & $99.17 \pm 1.01$ & $96.21 \pm 1.03$ & $-2.96 \pm 0.40$ & $<0.001$ \\
\hline WHR & $0.90 \pm 0.01$ & $0.89 \pm 0.01$ & $-0.01 \pm 0.00$ & $<0.001$ \\
\hline FPG (mg/dL) & $99.46 \pm 2.05$ & $97.33 \pm 1.67$ & $-2.13 \pm 1.54$ & 0.171 \\
\hline $\mathrm{HbA1c}(\%)$ & $5.63 \pm 0.07$ & $5.68 \pm 0.07$ & $0.05 \pm 0.04$ & 0.242 \\
\hline Insulin ( $\mu \mathrm{lU} / \mathrm{mL})$ & $12.51 \pm 0.90$ & $11.16 \pm 0.93$ & $-1.34 \pm 0.73$ & 0.071 \\
\hline HOMA-IR & $3.04 \pm 0.20$ & $2.71 \pm 0.24$ & $-0.33 \pm 0.18$ & 0.076 \\
\hline $\mathrm{TC}$ (mg/dL) & $194.69 \pm 4.42$ & $187.90 \pm 4.49$ & $-6.79 \pm 3.37$ & 0.048 \\
\hline $\mathrm{TG}(\mathrm{mg} / \mathrm{dL})$ & $153.40 \pm 14.60$ & $159.76 \pm 13.46$ & $6.35 \pm 11.22$ & 0.573 \\
\hline $\mathrm{HDL}-\mathrm{C}$ (mg/dL) & $52.48 \pm 1.59$ & $48.29 \pm 1.36$ & $-4.19 \pm 1.00$ & $<0.001$ \\
\hline LDL-C (mg/dL) & $123.63 \pm 3.83$ & $118.52 \pm 3.74$ & $-5.11 \pm 2.93$ & 0.086 \\
\hline Adiponectin ( $\mu \mathrm{g} / \mathrm{mL})$ & $9.25 \pm 4.62$ & $9.43 \pm 5.31$ & $0.18 \pm 3.11$ & 0.648 \\
\hline IL-10 (pg/mL) & $0.25 \pm 0.15$ & $0.33 \pm 0.51$ & $0.08 \pm 0.06$ & 0.173 \\
\hline $\ln (I L-10)$ & $-1.49 \pm 0.05$ & $-1.50 \pm 0.10$ & $-0.01 \pm 0.08$ & 0.926 \\
\hline
\end{tabular}

Values are presented as mean \pm standard error. $P$-values were calculated using Wilcoxon signed-rank tests.

IL-10, interleukin-10; BMI, body mass index; WC, waist circumference; WHR, waist to hip circumference ratio; $\mathrm{FPG}$, fasting plasma glucose; $\mathrm{HbA1c}$, glycosylated hemoglobin HOMA-IR, homeostasis model assessment-insulin resistance; TC, total cholesterol; TG, triglycerides; HDL-C, high-density lipoprotein cholesterol; LDL-C, low-density lipoprotein cholesterol. 

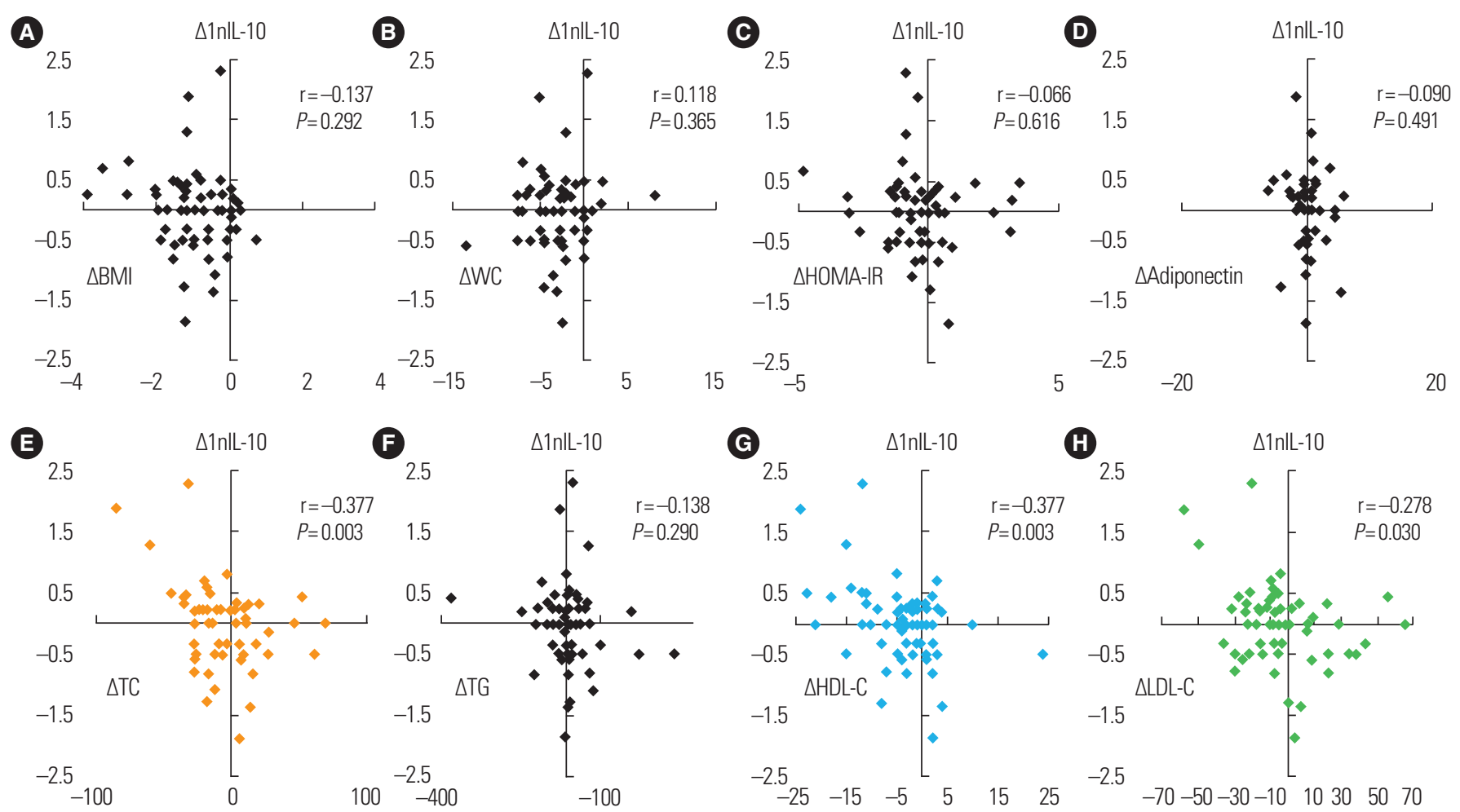

Figure 1. Correlations between changes in serum interleukin-10 (IL-10) concentration and changes in cardiometabolic risk factors: (A) BMI, (B) WC, (C) HOMA-IR, (D) Adiponectin, (E) TC, (F) TG, (G) HDL-C, (H) LDL-C. P-values were calculated by partial correlation analysis after adjustment for age. BMl, body mass index; WC, waist circumference; HOMA-IR, homeostasis model assessment-insulin resistance; TC, total cholesterol; TG, triglycerides; HDL-C, high-density lipoprotein cholesterol; LDL-C, low-density lipoprotein cholesterol.

were significantly lower after weight reduction $(P<0.05)$. However, the changes in IL-10 concentration were not significant. Correlations between changes in serum IL-10 and changes in cardiometabolic parameters are shown in Fig. 1. Changes in serum IL-10 concentration were significantly negatively correlated with changes in total cholesterol ( $\mathrm{r}=-0.377, P=0.003)$, HDL-C $(\mathrm{r}=-0.377, P=0.003)$, and low-density lipoprotein cholesterol (LDL-C; $r=-0.278, P=$ 0.030). However, there were no correlations between the changes in serum IL-10 concentration and changes in other cardiometabolic parameters.

\section{DISCUSSION}

The present study showed that changes in serum IL-10 during weight loss are negatively associated with changes in serum total cholesterol, HDL-C, and LDL-C but not with other cardiometabolic risk factors. The changes in lipid parameters during the program might be due to both weight reduction and use of orlistat.
However, since the difference in IL-10 concentration was not significant between baseline and post-intervention, the changes might be explained by a greater association with changes in lipid parameters rather than the effects of orlistat. In multiple linear regression analysis using the stepwise selection method, the $\beta$-coefficient for the relationship between changes in serum IL-10 and in serum total cholesterol was $-0.471(P<0.001)$. Most previous studies have shown IL-10 to have anti-inflammatory and anti-atherogenic properties, but recent studies have generated discrepant results. The significant relationships between changes in $\mathrm{IL}-10$ concentration and in both LDL-C and HDL-C in the present study might reflect the inconsistency in the literature regarding the association between serum IL-10 and outcomes of cardiovascular disease.

In a previous animal study, systemic overexpression of IL-10 lowered plasma LDL-C and very low-density lipoprotein cholesterol in LDL receptor knockout mice, potentially due to an effect on lipoprotein lipase activity. ${ }^{12}$ In another study, IL-10 deficiency led to higher LDL-C in apolipoprotein (apo)E knockout mice. ${ }^{13}$ 
Furthermore, treatment with PEGylated recombinant human IL-10 lowered total plasma cholesterol in cancer patients with hypercholesterolemia. ${ }^{14}$ These findings are consistent with those of the present study.

IL-10 inhibits tumor necrosis factor-alpha secretion by monocytes in humans, ${ }^{15}$ which has been shown to increase the production of cholesterol by increasing the activity of hydroxy-3-methylglutaryl-coenzyme A reductase, the rate-limiting enzyme in cholesterol synthesis. ${ }^{16}$ With regard to atherosclerosis, IL-10 plays a major role by inhibiting macrophage activation and matrix metalloproteinase, proinflammatory cytokines, and cyclooxygenase- 2 expression in lipid-loaded and activated macrophage foam cells. ${ }^{17}$ In addition, it enhances phagocytosis by liver-resident Kupffer cells, which lowers total plasma cholesterol concentration. ${ }^{14}$

A recent study has suggested that high circulating IL-10 might be a novel cause of dyslipidemia, leading to severe HDL deficiency. ${ }^{18}$ That study exhibited a significant negative relationship between IL-10 and HDL-C $\left(\mathrm{R}^{2}=-0.372\right)$ in patients with autoimmune lymphoproliferative syndrome, which is consistent with our findings. Lower lecithin cholesterol acyltransferase activity, lower apoAI synthesis and secretion in the intestine, and greater catabolism of $\mathrm{HDL}$ in response to high IL-10 concentration might be possible mechanisms by which IL-10 could contribute to a reduction in HDL-C. ${ }^{18}$

We did not identify significant correlations between changes in serum IL-10 and changes in other cardiometabolic parameters. However, serum IL-10 was significantly positively correlated with body weight $(r=0.335)$ and waist circumference $(r=0.320)$ in the study participants (data not shown). These findings are similar to those of a previous study in which serum IL-10 was higher in obese than in non-obese women. ${ }^{3}$ A recent study has shown low serum IL-10 in obese children with hypertriglyceridemia. ${ }^{19}$ However, we did not identify a correlation between changes in serum IL-10 and changes in serum triglyceride in the present study. Serum triglycerides increased slightly while HDL-C significantly decreased during the intervention. This could be explained by a heterogeneous response of serum triglycerides and consumption of a relatively lowfat and high-carbohydrate diet during the intervention. In addition, a recent meta-analysis of the effect of orlistat on plasma lipids reported that its use was associated with a slight reduction of plasma
HDL-C concentration in 19 of 36 trials. $^{20}$

The present study has several limitations. First, cause-and-effect relationships cannot be ascribed. Second, we cannot exclude the possibility of confounding factors affecting serum cholesterol and IL-10 concentrations. Third, the weight reduction in our study subjects was modest, potentially limiting the changes in serum $\mathrm{IL}$ 10 during the study period. We analyzed the post hoc differences in serum IL-10 separately in participants who lost weight and in those who did not ( $<2 \%$ reduction in body weight). However, no significant difference between the responders and non-responders was found. Additional mechanistic and large-scale epidemiologic studies are required to clarify the role of IL-10. In conclusion, we showed that changes in serum IL-10 negatively correlated with changes in total cholesterol, HDL-C, and LDL-C during a weight loss program.

\section{CONFLICTS OF INTEREST}

Ga Eun Nam has worked as an Associate Editor of the journal since 2020. However, she was not involved in the peer reviewer selection, evaluation, or decision process of this article. Otherwise, no other potential conflicts of interest relevant to this article were reported.

\section{ACKNOWLEDGMENTS}

This study was supported by the Korean Ministry of Education, Science, and Technology (grant no. 2017030666).

\section{AUTHOR CONTRIBUTIONS}

Study concept and design: all authors; acquisition of data: all authors; analysis and interpretation of data: all authors; drafting of the manuscript: SJK, JL, and GEN; critical revision of the manuscript: all authors; statistical analysis: all authors; study supervision: GEN.

\section{REFERENCES}

1. Mosser DM, Zhang X. Interleukin-10: new perspectives on 
an old cytokine. Immunol Rev 2008;226:205-18.

2. Mallat Z, Heymes C, Ohan J, Faggin E, Lesèche G, Tedgui A. Expression of interleukin-10 in advanced human atherosclerotic plaques: relation to inducible nitric oxide synthase expression and cell death. Arterioscler Thromb Vasc Biol 1999; 19:611-6.

3. Esposito K, Pontillo A, Giugliano F, Giugliano G, Marfella R, Nicoletti G, et al. Association of low interleukin-10 levels with the metabolic syndrome in obese women. J Clin Endocrinol Metab 2003;88:1055-8.

4. Straczkowski M, Kowalska I, Nikolajuk A, Krukowska A, Gorska M. Plasma interleukin-10 concentration is positively related to insulin sensitivity in young healthy individuals. Diabetes Care 2005;28:2036-7.

5. Jung SH, Park HS, Kim KS, Choi WH, Ahn CW, Kim BT, et al. Effect of weight loss on some serum cytokines in human obesity: increase in IL-10 after weight loss. J Nutr Biochem 2008;19:371-5.

6. Heeschen C, Dimmeler S, Hamm CW, Fichtlscherer S, Boersma E, Simoons ML, et al. Serum level of the anti-inflammatory cytokine interleukin-10 is an important prognostic determinant in patients with acute coronary syndromes. Circulation 2003;107:2109-14.

7. Zhu Y, Yang H, Diao Z, Li Y, Yan C. Reduced serum level of interleukin-10 is associated with cerebral infarction: a case-control and meta-analysis study. Mol Neurobiol 2016;53:2698-704.

8. Wilke CM, Wei S, Wang L, Kryczek I, Kao J, Zou W. Dual biological effects of the cytokines interleukin-10 and interferon- $\gamma$. Cancer Immunol Immunother 2011;60:1529-41.

9. Mälarstig A, Eriksson P, Hamsten A, Lindahl B, Wallentin L, Siegbahn A. Raised interleukin-10 is an indicator of poor outcome and enhanced systemic inflammation in patients with acute coronary syndrome. Heart 2008;94:724-9.

10. Mühl H. Pro-inflammatory signaling by il-10 and il-22: bad habit stirred up by interferons? Front Immunol 2013;4:18.

11. Matthews DR, Hosker JP, Rudenski AS, Naylor BA, Treacher DF, Turner RC. Homeostasis model assessment: insulin resis- tance and beta-cell function from fasting plasma glucose and insulin concentrations in man. Diabetologia 1985;28:412-9.

12. Von Der Thüsen JH, Kuiper J, Fekkes ML, De Vos P, Van Berkel TJ, Biessen EA. Attenuation of atherogenesis by systemic and local adenovirus-mediated gene transfer of interleukin-10 in LDLr-/- mice. FASEB J 2001;15:2730-2.

13. Caligiuri G, Rudling M, Ollivier V, Jacob MP, Michel JB, Hansson GK, et al. Interleukin-10 deficiency increases atherosclerosis, thrombosis, and low-density lipoproteins in apolipoprotein E knockout mice. Mol Med 2003;9:10-7.

14. Chan IH, Van Hoof D, Abramova M, Bilardello M, Mar E, Jorgensen B, et al. PEGylated IL-10 activates kupffer cells to control hypercholesterolemia. PLoS One 2016;11:e0156229.

15. Shin DI, Banning U, Kim YM, Verheyen J, Hannen M, Bönig $\mathrm{H}$, et al. Interleukin 10 inhibits TNF-alpha production in human monocytes independently of interleukin 12 and interleukin 1 beta. Immunol Invest 1999;28:165-75.

16. Memon RA, Grunfeld C, Moser AH, Feingold KR. Tumor necrosis factor mediates the effects of endotoxin on cholesterol and triglyceride metabolism in mice. Endocrinology 1993; 132:2246-53.

17. Han X, Boisvert WA. Interleukin-10 protects against atherosclerosis by modulating multiple atherogenic macrophage function. Thromb Haemost 2015;113:505-12.

18. Moraitis AG, Freeman LA, Shamburek RD, Wesley R, Wilson W, Grant CM, et al. Elevated interleukin-10: a new cause of dyslipidemia leading to severe HDL deficiency. J Clin Lipidol 2015;9:81-90.

19. Liu Y, Xu D, Yin C, Wang S, Wang M, Xiao Y. IL-10/STAT3 is reduced in childhood obesity with hypertriglyceridemia and is related to triglyceride level in diet-induced obese rats. BMC Endocr Disord 2018;18:39.

20. Sahebkar A, Simental-Mendía LE, Reiner Ž, Kovanen PT, Simental-Mendía M, Bianconi V, et al. Effect of orlistat on plasma lipids and body weight: a systematic review and meta-analysis of 33 randomized controlled trials. Pharmacol Res 2017; 122:53-65. 